\title{
Effect of Diet Change on Gut Microbiota: Observational Pilot Study of Four Urban Couples
}

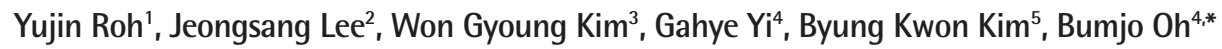 \\ ${ }^{1}$ Department of Medicine, Seoul National University College of Medicine, Seoul; Departments of ${ }^{2}$ Thoracic and Cardiovascular Surgery, ${ }^{3}$ Nutrition Service, and \\ ${ }^{4}$ Family Medicine, SMG-SNU Boramae Medical Center, Seoul; ${ }^{5}$ Omicspia Co., Ltd., Daejeon, Korea
}

\begin{abstract}
Background: Recent studies have focused on changes in gut microbiota following a dietary change. We identified how the distribution of gut microbiota changed when the dietary habits of young city dwellers improved using an intervention in which married couples shared the same dietary habits.

Methods: Four married couples in their 30s with irregular eating habits and sedentary lifestyles were asked whether they had any uncomfortable symptoms. A nutritionist advised them to reduce their intake of processed meats, carbonated beverages, and late-night snacks. After a 6-week intervention, subjects were asked whether they observed any changes in their symptoms. Their stool samples were collected before and after the intervention and analyzed to determine whether the gut microbiota had changed.

Results: After the dietary intervention, some subjective symptoms of the participants improved. Specifically, a subject who complained of frequent abdominal pain/diarrhea and one who complained of fatigue showed improvement in those symptoms. In addition, some subjects showed improvements in symptoms such as skin disease or constipation. Intestinal microorganisms between spouses who share the same dietary habits were found to be similar.

Conclusion: Improvements in eating habits can change the distribution of gut microbiota and alleviate various uncomfortable medical symptoms. Within married couples, the distribution of gut microbiota became similar when the spouses shared the same dietary habits. These results suggest a possible correlation between familylevel changes in eating habits and the health of all family members.
\end{abstract}

Key words: Diet therapy, Gastrointestinal microbiome, Urban population, Spouses
Received October 31, 2017

Reviewed November 10, 2017

Accepted November 17, 2017

*Corresponding author

Bumjo Oh

https://orcid.org/0000-0002-2468-0755

Department of Family Medicine, SMG-SNU Boramae Medical Center, 20 Boramae-ro 5-gil, Dongjak-gu, Seoul 07061, Korea

Tel: +82-2-870-2682

Fax: +82-2-831-0714

E-mail: bo39@snu.ac.kr

The first two authors contributed equally to this study.

\section{INTRODUCTION}

Chronic conditions such as type 2 diabetes, autoimmune diseases, cardiovascular disease, metabolic syndrome, and obesity are widely considered to be associated with unhealthy lifestyles and behaviors. ${ }^{1}$ Thus, lifestyle modifications, including stress management, emotional well-being, positive social relationships, smoking cessation, balanced diet, regular physical activity (PA), and moderate consumption of alcohol ${ }^{2-5}$, are important in preventing and treating those diseases.

Meanwhile, interest has been growing in indigenous microbial communities and the host environment that they inhabit. Microbes that inhabit human bodies benefit the entire host-microbe system by supplying crucial ecosystem services. They produce important resources, convert nutrients biologically, and protect their host from pathogenic microbes. ${ }^{6}$ Therefore, the disruption of the beneficial functions of microbiota can lead to disease. Recently, many studies have suggested a correlation between microbiota and some chronic illnesses, such as inflammatory bowel disease, obesity, type 1 diabetes, and pulmonary diseases. ${ }^{7-11}$

As an easily modifiable lifestyle factor, diet has substantial effects on human health by modifying intestinal microbiota. ${ }^{12,13}$ Many data 
suggest the importance of diet in establishing human intestinal microbiota. ${ }^{14,15}$ The consumption of various nutrients affects the structure of the microbial community and provides substrates for microbial metabolism. ${ }^{16}$ The microbiota can produce molecules absorbed by the host and can affect many important physiological processes. ${ }^{16,17}$ Functional studies in animal models, as well as descriptive association studies in humans, provide evidence for the role of diet in disease pathogenesis through its effects on intestinal microbes. ${ }^{16}$

Couples who live together share their lifestyles. They tend to eat similar foods, do exercise together, and enjoy leisure activities together. As time goes by, their physical condition might become very similar to each other, and they become susceptible to similar diseases. ${ }^{18}$ Because diet can alter gut microbiota, we analyzed the intestinal microbiome of eight people (four married couples) to observe changes in microbiota following a dietary intervention. We also determined how similar changes in individuals were to those in their partner. Participants were asked to maintain a well-balanced and healthy diet for 6 weeks.

\section{METHODS}

\section{Participants}

Four Korean couples (eight participants) with skin diseases (psoriasis, atopic dermatitis, etc.) or gastrointestinal symptoms (diarrhea, constipation, etc.) were recruited for this research. This study was approved by the Institutional Review Board of SMG-SNU Boramae Medical Center (IRB No. 26-2016-141), and written informed consent was obtained from all participants before their admission to the protocol. Symptoms and characteristics of the four couples were evaluated, and we carried out an anthropometric investigation and nutritional survey to establish participants' nutritional status and dietary lifestyles. Their fecal samples were also collected. For 6 weeks, the participants submitted to a dietary intervention to reduce their intake of processed meat, carbonated beverages, and late-night snacks, while maintaining a well-balanced healthy diet. The couples were asked to eat at least one meal together every day. After the intervention, their fecal samples were collected again, and each individual's gut microbiota were analyzed using a highthroughput sequencing technique. Participants were also asked to report any changes in their symptoms.

\section{Characteristics of participants}

Social history, physical activity, and mental health

We interviewed participants to learn their occupations and whether they had any uncomfortable health problems. We also conducted a survey in written form to ask participants if they had ever smoked. If a participant answered "yes," we asked how long they had smoked and how many cigarettes they had smoked each day. Through the survey, we asked participants if they drank alcohol. If a participant answered "yes," we asked how often they drank and how much they drank per occasion.

We used the International Physical Activity Questionnaire to assess PA in three domains. ${ }^{19}$ The specific types of activity assessed are walking, moderate-intensity activities (moderate PA), and vigorous intensity activities (vigorous PA). Frequency (measured in days per week) and duration (time per day) are collected separately for each specific type of activity. The volume of activity can be computed by weighting each type of activity by its energy requirements defined in metabolic equivalents (METs; multiples of the resting metabolic rate) to yield a score in MET-minutes. A MET-minute is computed by multiplying the MET score by the minutes performed: walking = $3.3 \mathrm{METs}$, moderate $\mathrm{PA}=4.0 \mathrm{METs}$, and vigorous $\mathrm{PA}=$ 8.0 METs.

We used the Korean-translated Brief Encounter Psychosocial Instrument (BEPSI-K) to assess the stress level of participants. BEPSI is a stress evaluation scale developed in 1988 by Frank and Zyzanski. ${ }^{20}$ In 1996, the Korean version of BEPSI, BEPSI-K was developed by Yim et al. ${ }^{21}$ It contains five questions and is designed to assess stress during the past month. Each item is rated on a scale of 0-4 points, and an average of 2.4 points or more is considered to be stressful. Because the BEPSI contains only a few simple questions, it can be used conveniently in primary care situations. ${ }^{22}$

We used the Patient Health Questionnaire-9 (PHQ-9) to screen for depression. The PHQ-9 incorporates the Diagnostic and Statistical Manual of Mental Disorders-IV depression diagnostic criteria and other leading major depressive symptoms into a brief self-report tool. The tool rates the frequency of the symptoms that factor into the scoring severity index. PHQ scores of 10 or more had a sensitivity of $88 \%$ and a specificity of $88 \%$ for major depression. PHQ-9 scores of 5, 10, 15, and 20 represent mild, moderate, moderately severe, and severe depression, respectively. ${ }^{23}$ 


\section{Body measurement and assessing obesity}

We measured the height and body weight of participants. Their body mass index (BMI), body fat percentage, and basal metabolic rate (BMR) were assessed using eight-polar bioelectrical impedance (InBody 3.0; Biospace, Seoul, Korea).

\section{Dietary assessment and intervention}

Dietary habits and diet quality

To evaluate the dietary habits of participants, we asked three questions about bad eating habits: "How often do you drink carbonated drinks?", "How often do you eat out?", and "How often do you eat fast food?" The first item was rated as $0-3$ points; the second item was rated as 0-2 points, and the last item was rated as $0-1$ point. We then summed all points.

We used the Recommended Food Score (RFS), a food-frequency questionnaire based on reported consumption of foods containing high amounts of antioxidant nutrients, to measure overall diet quality. Because current dietary guidelines emphasize the consumption of whole grains, legumes, vegetables, fruits, fish, dairy products, nuts, and tea, participants received 1 point for each recommended food they reported consuming at least weekly. A total of 46 foods or food groups corresponding to recommended food groups were selected. We also used the "daily frequency of meals" to calculate the RFS. Thus, the maximum possible score was 47 , with scores greater than 36 points indicating a high-quality diet. ${ }^{24}$

\section{Diet related health risk appraisal}

We used a tool called the diet related health risk appraisal to assess the risk of chronic diseases based on individual dietary habits.

\section{Dietary assessment}

Before nutritional counseling, each participant wrote 3-day food record. For the analysis of the nutrients (carbohydrates, protein, fat) in the food consumed, we used the CAN-Pro 4.0 (Computer Aided Nutrition Analysis Program) from the Korean Nutrition Society. A skilled clinical dietitian diagnosed nutritional problems based on the dietary assessment and conducted individual nutritional counseling.

\section{Fecal sample collection and preparation}

Fecal samples, which were taken at the participant's home at the beginning and end of the program, were collected and stored at $-80^{\circ} \mathrm{C}$ until analysis.

\section{DNA extraction and pyrosequencing}

Metagenomic DNA was extracted from the fecal samples using a FastDNA SPIN extraction kit (MP Biomedicals, Santa Ana, CA, USA). The $16 \mathrm{~S}$ rRNA gene (target region V1-V3) was amplified with barcoded primers using a C1000 Touch thermal cycler (BioRad, Hercules, CA, USA). Amplified products were purified using the QIAquick PCR purification kit (Qiagen, Valencia, CA, USA) and quantified using the PicoGreen dsDNA assay kit (Invitrogen, Carlsbad, CA, USA). Equimolar concentrations of samples were pooled and sequenced on a Roche/454 GS junior system (Roche Sequencing, Pleasanton, CA, USA) according to the manufacturer's instructions.

\section{Pyrosequencing analysis}

Sequences produced by the pyrosequencer were analyzed according to previous descriptions. Briefly, sequences for each sample were sorted using a unique barcode, and low-quality reads (average quality score $<25$ or read length $<300 \mathrm{bp}$ ) were removed. Primer sequences were trimmed by the HMM-search program of the HMMER 3.0 package (http://hmmer.org/). Trimmed sequences were clustered by $97 \%$ similarity, and representative sequences in each cluster were selected to identify their taxonomic positions. The taxonomic assignments of reads were conducted using the RDPclassifier with SILVA database and BLAST algorithm against EzTaxon-e database. Chimera sequences were detected and removed for further analysis using UCHIME. The diversity indices were calculated using the Mothur program (Mothur, Ann Arbor, MI, USA) after the read number in each sample had been normalized. The pyrosequencing reads obtained from this study are available in the European Molecular Biology Laboratory Sequence Read Archive database under study number.

\section{Statistical analysis}

Statistical analyses were performed using the STATA version 12.0 (Stata Corp., College Station, TX, USA). The changes in fecal mi- 
crobiota were set as the dependent variable, and the body measurements and survey results were set as the discriminatory variables. All survey and examination results were analyzed using the chi-square test and descriptive statistics to determine the frequency, percentages, and averages. The Pearson correlation method was used to confirm whether the Firmicutes to Bacteroidetes ratio was related to anthropometric data, including BMI. The $P$-value was determined using the Kruskal-Wallis H-test and Mann-Whitney rank-sum test, and a post-hoc test was performed using the Tukey-Kramer method. Multiple test corrections were made using the Benjamini-Hochberg false discovery rate. Results with $P$-values $<0.05$ (two-tailed) were considered statistically significant.

\section{RESULTS}

\section{Characteristics of participants}

Table 1 summarizes the characteristics of the subjects, including their lifestyles.

\section{Social history, physical activity, and mental health}

Participant 1-H (husband) is a government employee with mild gastritis. Participant 1-W (wife) is a housewife with severe constipation. She also has mild gastritis and allergic rhinitis. Participant $2-\mathrm{H}$ is a building materials dealer with frequent diarrhea. He has smoked one pack a day for 10 years and drinks 5 days a week, five or six glasses per day. Participant 2-W is a housewife who frequently has gas in the bowels. She drinks 2 days a week, one or two glasses per day. Participant 3-H is a computer programmer who has suffered from atopic dermatitis for 17 years. Participant 3-W is a housewife who frequently suffers from enteritis. Participant 4-H is an IT (information technology) company employee with no underlying disease. He has smoked one pack a day for 10 years and drinks 5 days a week, seven or nine glasses per day. Participant 4-W is a housewife with psoriasis.

Based on MET-minutes scores, we assessed each participant's level of PA. Participants 1-H, 2-W, and 4-W were classified as "inactive." Participants $2-\mathrm{H}$ and 3-H were classified as "health enhancing physical activity active." Participants 1-W, 3-W, and 4-H were classified as "minimally active."

Using BEPSI-K, no participant felt severe stress. On the other hand, according to their PHQ-9 scores, participants 2-W and 4-H had mild depression. The other participants had no depression.

Table 1. Characteristics of participants

\begin{tabular}{|c|c|c|c|c|c|c|c|c|}
\hline Variable & $1-\mathrm{H}$ & $1-W$ & $2-\mathrm{H}$ & $2-W$ & $3-\mathrm{H}$ & $3-W$ & $4-\mathrm{H}$ & $4-W$ \\
\hline Age (yr) & 36 & 35 & 39 & 35 & 34 & 29 & 33 & 33 \\
\hline Height (cm) & 172 & 160 & 173 & 167 & 169 & 159 & 180 & 173 \\
\hline Weight (kg) & 72.6 & 53.5 & 77.5 & 58.3 & 71.7 & 43.4 & 80.4 & 52.3 \\
\hline $\mathrm{BMI}\left(\mathrm{kg} / \mathrm{m}^{2}\right)$ & 24.5 & 20.9 & 25.9 & 20.9 & 25.1 & 17.2 & 24.8 & 17.5 \\
\hline Body fat mass (\%) & 23.4 & 29.1 & 20.4 & 27.0 & 26.9 & 24.2 & 24.0 & 17.0 \\
\hline BMR (kcal) & 1,571 & 1,189 & 1,703 & 1,290 & 1,502 & 1,081 & 1,691 & 1,307 \\
\hline Medication & - & - & - & - & - & - & - & - \\
\hline Past medical history & $\begin{array}{l}\text { Mild gastritis, } \\
\text { abdominal } \\
\text { obesity }\end{array}$ & $\begin{array}{l}\text { Constipation, mild } \\
\text { gastritis, aller- } \\
\text { gic rhinitis }\end{array}$ & Frequent diarrhea & $\begin{array}{l}\text { Abdominal gas, } \\
\text { bowel disten- } \\
\text { tion }\end{array}$ & Atopic dermatitis & $\begin{array}{l}\text { Frequent enteritis, } \\
\text { frequent diar- } \\
\text { rhea }\end{array}$ & - & Psoriasis \\
\hline Smoking & - & - & Ex-smoker, $10 \mathrm{PY}$ & - & - & - & Ex-smoker, $10 \mathrm{PY}$ & - \\
\hline Alcohol & - & - & $\begin{array}{c}\text { 5-6 glass/day, } \\
5 \text { day/wk }\end{array}$ & $\begin{array}{c}\text { 1-2 glass/day, } \\
2 \text { day/wk }\end{array}$ & - & - & $\begin{array}{c}\text { 7-9 glass/day, } \\
5 \text { day/wk }\end{array}$ & - \\
\hline IPAO (MET-min/wk) & 66 & 756 & 3,590 & 66 & 3,924 & 2,076 & 990 & 231 \\
\hline BEPSI-K (min, 0; max, 4) & 0.6 & 0 & 0.6 & 1 & 0.4 & 1.4 & 0.8 & 3 \\
\hline PHQ-9 (min, 0; max, 27) & 0 & 1 & 3 & 5 & 3 & 2 & 7 & 3 \\
\hline Sleep duration (hr) & 7 & 7 & 7 & 6 & 5.5 & 8 & 7 & 7 \\
\hline $\operatorname{Diet}(\min , 0 ; \max , 6)$ & 2 & 0 & 1 & 3 & 5 & 3 & 5 & 1 \\
\hline $\mathrm{RFS}$ (min, 0; max, 47) & 28 & 22 & 9 & 18 & 17 & 18 & 20 & 4 \\
\hline
\end{tabular}

Each couple is numbered, using $\mathrm{H}$ and $\mathrm{W}$ to indicate husband and wife, respectively.

BMI, body mass index; BMR, basal metabolic rate; PY, pack-year; IPAQ, International Physical Activity Questionnaire; MET, metabolic equivalent; BEPSI-K, Korean-translated Brief Encounter Psychosocial Instrument; min, minimum; max, maximum; PHQ-9, Patient Health Questionnaire-9; RFS, Recommended Food Score. 


\section{Body measurement and assessing obesity}

The World Health Organization Asian BMI risk cut points establish the following three BMI categories: $18.5-22.9 \mathrm{~kg} / \mathrm{m}^{2}$ (normal weight), $23-27.5 \mathrm{~kg} / \mathrm{m}^{2}$ (overweight), and $\geq 27.5 \mathrm{~kg} / \mathrm{m}^{2}$ (obese). All male participants are overweight. Participants 3-W and 4-W have BMI scores below the normal range, which means they are underweight. Participants 1-W and 2-W have BMI scores in the normal range.

\section{Evaluation of nutritional status and dietary intervention Dietary habit and diet quality}

Using the RFS, no participants began the study with a good quality diet (Table 1).

\section{Diet related health risk appraisal}

The risk of chronic diseases was assessed by diet related health risk appraisal (Supplementary Table 1). Participants 1-H, 2-H, 3-H, and 4-H have a very high risk of hypercholesterolemia; participant $1-\mathrm{H}$ has a high risk of hypertension; participant $4-\mathrm{H}$ has a moderate risk of diabetes mellitus; participants $3-\mathrm{H}$ and $4-\mathrm{H}$ have a high risk of obesity, and participants 2-W, 3-H, and 4-H have a moderate risk of abdominal obesity.

\section{Dietary assessment}

Nutritional problems indicated in the dietary assessment and the causes of each problem were assessed (Supplementary Tables 2 and 3$)$.

\section{Comparison of gut microbiota distribution before and after the dietary intervention \\ Phylum level}

In all samples, the highest percentage of gut microbiota were $\mathrm{Bac}$ teroidetes and Firmicutes. Veillonellaceae, Ruminococcaceae, and Lachnospiraceae are the main microorganisms in Bacteroidetes, and Rikenellaceae, Prevotellaceae, and Bacteroidaceae are the main microorganisms in Firmicutes. The Firmicutes to Bacteroidetes ratio is known to correlate with obesity: the larger that ratio, the greater the likelihood of obesity.

Comparing the results before and after the dietary interventions, most of the samples changed from their original state. The Firmicutes to Bacteroidetes ratio decreased in participants 1-H, 1-W, 3-H, 3-W, and 4-W and increased in participants 2-H, 2-W, and 4-H. We also used the Pearson correlation method to determine whether the anthropometric values correlate with the Firmicutes to Bacteroidetes ratio. Overall, we found no correlation between that ratio and body mass, body fat percent, BMI, BMR, or eating habits. However, when analyzed separately for male subjects, body fat mass and the Firmicutes to Bacteroidetes ratio showed a negative but nonsignificant correlation of $-0.91(P=0.089)$, and BMR and the Firmicutes to Bacteroidetes ratio showed a strong positive correlation of $0.96(P=0.037)$. Nevertheless, it is difficult to confirm the statistical significance of that correlation because the number of samples is only four, and it was not observed when the women were analyzed separately (Table 2).

In participants 1-H, 3-H, 3-W, and 4-W, Bacteroidetes increased significantly and Firmicutes decreased. On the other hand, in participants $2-\mathrm{H}$ and 4-H, Firmicutes increased significantly and Bacteroidetes decreased. The largest change occurred in participants 3- $\mathrm{H}$ and 4-W. In participant 4-W, Firmicutes accounted for $97.5 \%$ of the microbiota before the intervention. After the dietary control, Firmicutes decreased to $43.3 \%$, and Bacteroidetes that were not present before became $60.4 \%$ of the microbiota. In participant 3-H, Firmicutes accounted for $50 \%$ and Proteobacteria accounted for $34.8 \%$ before the intervention. After the dietary control, Firmicutes and Proteobacteria decreased to $23 \%$ and $0.05 \%$, respectively, and Bacteroidetes accounted for $76.5 \%$ of the microbiota. Proteobacteria are commonly observed in patients with diseases such as inflammatory bowel disease and are known to cause inflammation (Supplementary Fig. 1).

Table 2. Correlation between Firmicutes to Bacteroidetes ratio and anthropometric data

\begin{tabular}{llcccccc}
\hline $\begin{array}{c}\text { Sample } \\
\text { ID }\end{array}$ & Sex & $\begin{array}{c}\text { Weight } \\
(\mathrm{kg})\end{array}$ & $\begin{array}{c}\text { Bodyfat } \\
\text { mass } \\
(\%)\end{array}$ & $\begin{array}{c}\text { BMl } \\
\left(\mathrm{kg} / \mathrm{m}^{2}\right)\end{array}$ & $\begin{array}{c}\text { BMR } \\
(\mathrm{kcal})\end{array}$ & RFS & $\begin{array}{c}\text { Bacte- } \\
\text { roidetes/ } \\
\text { Firmicutes } \\
(\%)\end{array}$ \\
\hline 1-H & Male & 72.6 & 23.4 & 24.5 & 1,571 & 28 & 47.74 \\
1-W & Female & 53.5 & 29.1 & 20.9 & 1,189 & 22 & 108.25 \\
2-H & Male & 77.5 & 20.4 & 25.9 & 1,703 & 9 & 118.09 \\
2-W & Female & 58.3 & 27.0 & 20.9 & 1,290 & 18 & 50.46 \\
3-H & Male & 71.7 & 26.9 & 25.1 & 1,502 & 17 & 15.22 \\
3-W & Female & 43.4 & 24.2 & 17.2 & 1,081 & 18 & 106.22 \\
4-H & Male & 80.4 & 24.0 & 24.8 & 1,691 & 20 & 83.47 \\
4-W & Female & 52.3 & 17.0 & 17.5 & 1,307 & 4 & 0 \\
\hline
\end{tabular}

BMI, body mass index; BMR, basal metabolic rate; RFS, Recommended Food Score. 


\section{Genus level}

At the genus level, the samples containing Bacteroides as the major portion were 1-H (before the intervention [bef]), 1-H (after the intervention [aft]), 1-W (bef), 1-W (aft), 3-H (aft), 4-H (bef), and 4-H (aft). The samples containing Prevotella as the major portion were 2-H (bef), 2-W (aft), 3-W (bef), 3-W (aft), and 4-W (aft). In the 2-H (aft) sample, Ruminococcus (Ruminococcaceae) accounted for the major portion.

Prevotella, which is commonly observed in people who eat a lot of vegetable fiber, increased in participants 2-W, 3-W, and 4-W after the dietary control. On the other hand, in participant 2-H, it decreased. Overall, the Prevotella group showed an increase from $13.57 \%$ to $18.90 \%$, but there was no statistical significance because of individual differences. Participants 1-H, 1-W, 3-H, and 4-W saw an increase in Bacteroides, which are known to be common in people who consume a lot of meat.

\section{Species level}

Each sample shows a unique distribution of various species. Clostridium tertium, which accounted for $85.4 \%$ in sample 4-W (bef), was not detected at all in sample 4-W (aft). C. tertium is an opportunistic infectious microorganism that can cause bacteremia, meningitis, pyogenic arthritis, gastroenteritis, and pneumonia. In sample 4-W (aft), the AJ315483_s strain, which belongs to the genus Prevotella, was the dominant strain.

On the other hand, in participant 3-H, Escherichia coli accounted for $28.3 \%$ of the microbiota before the dietary intervention and was only about $0.05 \%$ after the intervention. In contrast, Alistipes shahii, a Bacteroidetes bacterium, showed a dominance of $29.9 \%$. In addition, the distribution of Lactobacillus, which is known to be beneficial, increased from $0.17 \%$ to $0.32 \%$ after dietary control. The distribution of the Clostridium and Escherichia groups, which are known to be harmful, decreased from $12.9 \%$ to $0.4 \%$ and from $3.6 \%$ to $0.05 \%$, respectively.

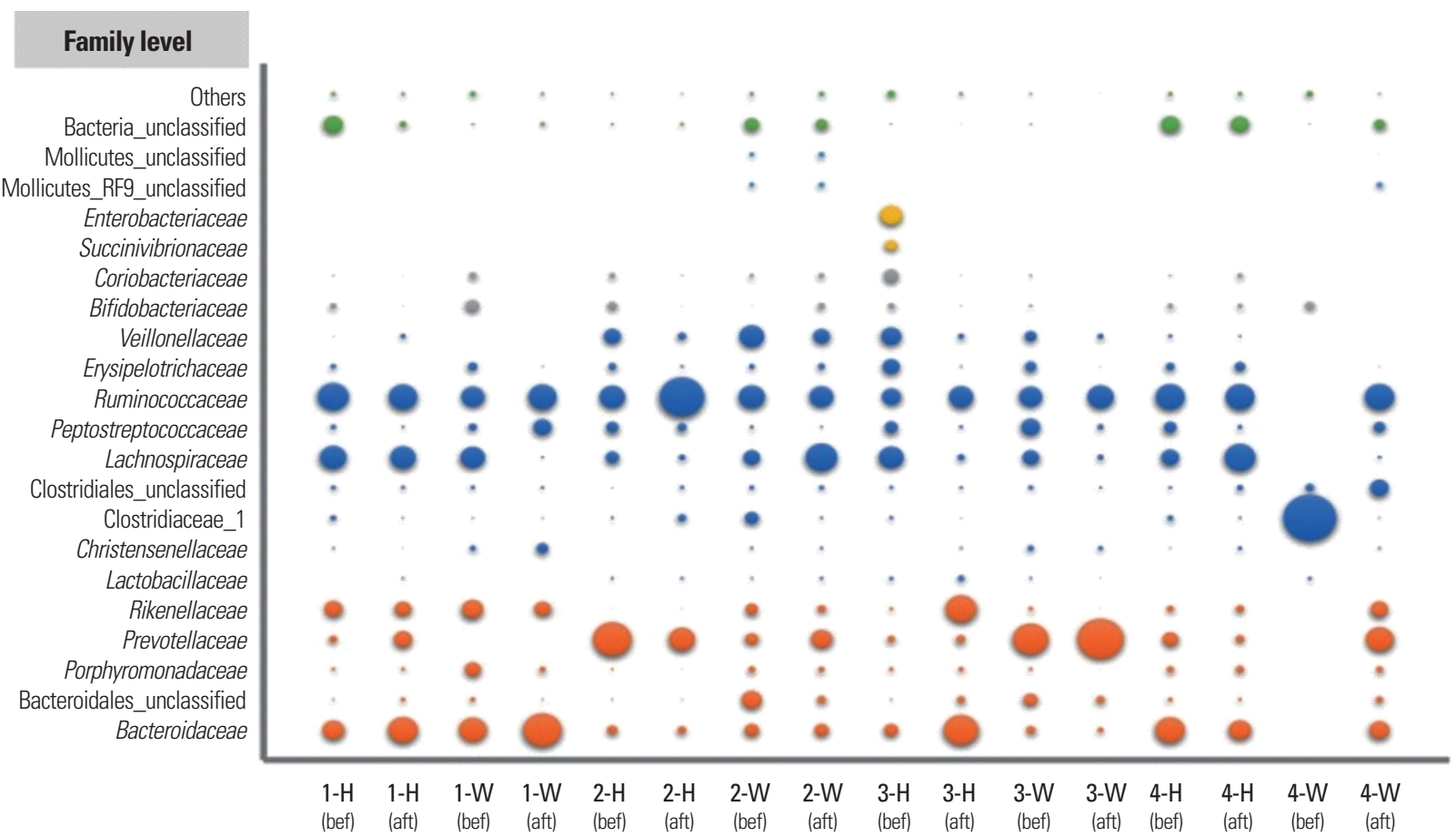

- Bacteroidetes

- Firmicutes

- Actinobacteria

- Proteobacteria

- Tenericutes

- Others

\section{Phylum level}

Figure 1. Microbial profiling chart. The size of the circles indicates the relative abundance of each family in the samples, and the colors indicate the phylum. Each couple is numbered, using $\mathrm{H}$ and $\mathrm{W}$ to indicate husband and wife, respectively. bef, before the intervention; aft, after the intervention. 


\section{Comparison of gut microbiota distribution between spouses}

The results from analyzing each individual's gut microbiota at the phylum and family levels are shown in Fig. 1. After the diet intervention, the overall distribution of intestinal microbiota in married couples became more similar, as is especially evident in couples 3-H/3-W, and 4-H/4-W (Fig. 1).

Enterobacteriaceae, Succinivibrionaceae, and Coriobacteriaceae were observed only in participant 3-H before the intervention. After the intervention, those bacterial colonies disappeared almost completely. Bacterial colonies belonging to the phylum Firmicutes (Veillonellaceae, Erysipelotrichaceae, Ruminococcaceae, Peptostreptococcaceae, and Lachnospiraceae) were about equally distributed in participant 3-H (bef), whereas afterward, Ruminococcaceae comprised a significant portion, and the rest of the bacterial colonies decreased. Similar changes in the composition of the phylum Firmicutes are also clear in participant 3-W.

Of all the participants, participant 4-W underwent the largest change. Participant 4-W had been taking antibiotics before participating in this research, which is evident in the large proportion of Clostridaceae in 4-W (bef). Sample 4-W (aft) showed a significant growth in Firmicutes, Bacteroidetes, and bacteria unclassified. This bacterial distribution closely resembles that of participant 4-W's spouse 4-H.

\section{Changes in subjective health status after the dietary intervention}

After the dietary interventions, participants 3-W and 4- $\mathrm{H}$ indicated that their symptoms had alleviated. Participant 3-W, who had frequent enteritis and diarrhea, replied that she had less frequent events of diarrhea after the intervention. Participant 4-H experienced an improvement in fatigue and loss of breath when engaging in physical activities such as climbing stairs.

\section{DISCUSSION}

Following a 6-week dietary intervention with four married couples in an urban area, the composition of gut microbiota tended to change in a similar manner between spouses. Moreover, after the intervention, the subjective health status of some participants had improved.

A recent study considered the effects of a gut microbiota-targeted dietary intervention. ${ }^{25}$ Xiao et al. ${ }^{25}$ designed a dietary scheme based on whole grains, traditional Chinese medicinal foods, and prebiotics (WTP diet) to meet human nutritional needs and balance gut microbiota. Obese volunteers completed a self-controlled clinical trial consisting of a 9-week intervention on the WTP diet followed by a 14-week maintenance period. Pyrosequencing of fecal samples showed that the phylotypes related to endotoxin-producing pathogens from Enterobacteriaceae and Desulfovibrionaceae decreased significantly, whereas those related to gut barrier-protecting bacteria from Bifidobacteriaceae increased. Those results suggest that modulation of the gut microbiota via dietary intervention could enhance the intestinal barrier integrity, reduce the circulating antigen load, and relieve the chronic inflammation that underlies metabolic syndrome. ${ }^{25}$ That study is similar to our study in its use of a dietary intervention, fecal sample collection, and analysis of gut microbiota with pyrosequencing. However, Xiao et al..$^{25}$ excluded participants with various pathologic conditions and used a stricter dietary intervention with three ready-to-use food formulas. In addition, they examined the effects of their intervention using changes in the biomarkers along the pathway that likely connects gut microbiota to the pathogenesis of obesity, rather than focusing on subjective improvement in symptoms.

Clearly, the results from only eight participants do not guarantee that all the described effects result from the dietary intervention. Also, the characteristics of the participants were heterogeneous, and all participants had different clinical symptoms. Moreover, we didn't control for the other daily activities of participants, including PA and lifestyle. Furthermore, we did only dietary consultation rather than regulating the exact foods eaten by participants. Therefore, it is difficult to determine whether the changes in intestinal microbiology were caused by a restriction of calorie intake or a change in the protein to carbohydrate ratio in the diet.

This study is one of many about intestinal microorganisms. It suggests the possibility of alleviating respiratory and gastrointestinal symptoms, as well as general conditions, by improving patients' eating habits. In addition, rather than just focusing on individual changes, we compared changes between spouses, thereby showing that improving family eating habits can have positive health effects 
on all family members. With a greater number of subjects and more sophisticated study design, it will be possible to identify whether a real causal relationship exists among dietary interventions, changes in gut microbiota, and improved health status.

\section{CONFLICTS OF INTEREST}

The authors declare no conflict of interest.

\section{SUPPLEMENTARY MATERIALS}

Supplementary Table 1. Diet related health risk appraisal. Supplementary Table 2. Nutritional problems of participants. Supplementary Table 3. Daily calorie intake from foods. Supplementary Figure 1. Microbiota distribution double pie chart. They can be found via https://doi.org/10.7570/jomes.2017.26. 4.257.

\section{REFERENCES}

1. Minich DM, Bland JS. Personalized lifestyle medicine: relevance for nutrition and lifestyle recommendations. ScientificWorldJournal 2013;2013:129841.

2. Roberts CK, Barnard RJ. Effects of exercise and diet on chronic disease. J Appl Physiol (1985) 2005;98:3-30.

3. Umberson D, Montez JK. Social relationships and health: a flashpoint for health policy. J Health Soc Behav 2010;51 Suppl: S54-66.

4. Turner J, Kelly B. Emotional dimensions of chronic disease. West J Med 2000;172:124-8.

5. Gritz ER, Vidrine DJ, Fingeret MC. Smoking cessation a critical component of medical management in chronic disease populations. Am J Prev Med 2007;33(Suppl 6):S414-22.

6. Young VB. The role of the microbiome in human health and disease: an introduction for clinicians. BMJ 2017;356:j831.

7. Zipris D. The interplay between the gut microbiota and the immune system in the mechanism of type 1 diabetes. Curr Opin Endocrinol Diabetes Obes 2013;20:265-70.

8. Round JL, Mazmanian SK. The gut microbiota shapes intestinal immune responses during health and disease. Nat Rev Im- munol 2009;9:313-23.

9. Ley RE, Turnbaugh PJ, Klein S, Gordon JI. Microbial ecology: human gut microbes associated with obesity. Nature 2006;444: 1022-3.

10. Clemente JC, Ursell LK, Parfrey LW, Knight R. The impact of the gut microbiota on human health: an integrative view. Cell 2012;148:1258-70.

11. Trompette A, Gollwitzer ES, Yadava K, Sichelstiel AK, Sprenger N, Ngom-Bru C, et al. Gut microbiota metabolism of dietary fiber influences allergic airway disease and hematopoiesis. Nat Med 2014;20:159-66.

12. Claesson MJ, Jeffery IB, Conde S, Power SE, O’Connor EM, Cusack S, et al. Gut microbiota composition correlates with diet and health in the elderly. Nature 2012;488:178-84.

13. Jeffery IB, O'Toole PW. Diet-microbiota interactions and their implications for healthy living. Nutrients 2013;5:234-52.

14. Flint HJ, Scott KP, Louis P, Duncan SH. The role of the gut microbiota in nutrition and health. Nat Rev Gastroenterol Hepatol 2012;9:577-89.

15. Flint HJ. The impact of nutrition on the human microbiome. Nutr Rev 2012;70 Suppl 1:S10-3.

16. Albenberg LG, Wu GD. Diet and the intestinal microbiome: associations, functions, and implications for health and disease. Gastroenterology 2014;146:1564-72.

17. Holmes E, Li JV, Marchesi JR, Nicholson JK. Gut microbiota composition and activity in relation to host metabolic phenotype and disease risk. Cell Metab 2012;16:559-64.

18. Jurj AL, Wen W, Li HL, Zheng W, Yang G, Xiang YB, et al. Spousal correlations for lifestyle factors and selected diseases in Chinese couples. Ann Epidemiol 2006;16:285-91.

19. Lee PH, Macfarlane DJ, Lam TH, Stewart SM. Validity of the international physical activity questionnaire short form (IPAQSF): a systematic review. Int J Behav Nutr Phys Act 2011;8: 115 .

20. Frank SH, Zyzanski SJ. Stress in the clinical setting: the brief encounter psychosocial instrument. J Fam Pract 1988;26:533-9.

21. Yim JH, Bae JM, Choi SS, Kim SW, Hwang HS, Huh BY. The validity of modified Korean-translated BEPSI (brief encounter psychosocial instrument) as instrument of stress measurement in outpatient clinic. J Korean Acad Fam Med 1996;17:42-53. 
22. Shin HC. Measuring stress with questionnaires. J Korean Med Assoc 2013;56:485-95.

23. Kroenke K, Spitzer RL, Williams JB. The PHQ-9: validity of a brief depression severity measure. J Gen Intern Med 2001; 16:606-13.

24. Kim JY, Yang YJ, Yang YK, Oh SY, Hong YC, Lee EK, et al.
Diet quality scores and oxidative stress in Korean adults. Eur J Clin Nutr 2011;65:1271-8.

25. Xiao S, Fei N, Pang X, Shen J, Wang L, Zhang B, et al. A gut microbiota-targeted dietary intervention for amelioration of chronic inflammation underlying metabolic syndrome. FEMS Microbiol Ecol 2014;87:357-67. 